\title{
Quantification of Hydroxyl Groups from Alumina in Aluminosilicate Materials by Adsorption Isotherms
}

\author{
Allyson Leandro Rodrigues dos Santos, Rosana Maria Nascimento de Assunção and \\ Anizio Marcio de Faria* \\ Instituto de Ciências Exatas e Naturais do Pontal, Universidade Federal de Uberlândia \\ Rua Vinte, 1600, 38304-402, Ituiutaba, MG, Brazil.
}

Graphical Abstract

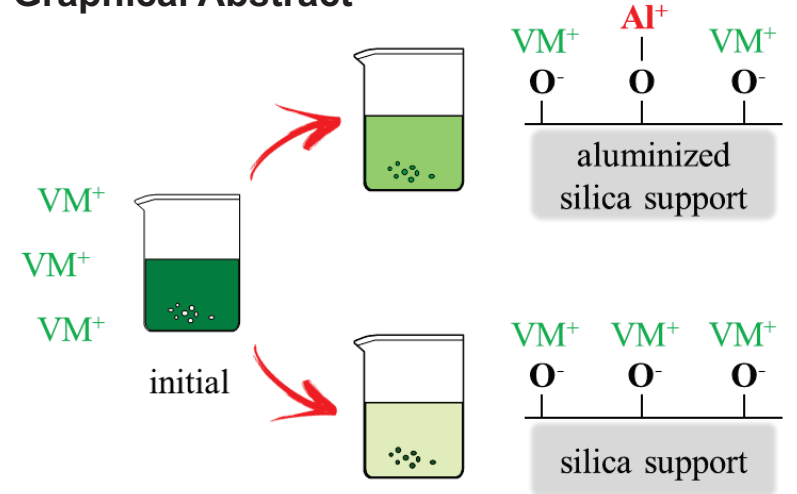

Determination of $\mathrm{Al}-\mathrm{OH}$ content in aluminized silica by the removal of malachite green from aqueous solution under controlled $\mathrm{pH}$ condition.
A simple and accessible method without the use of expensive and specific reagents is presented in this work for the quantification of hydroxyl groups from alumina in aluminized silica. The method is based on the differential interaction of a dye on bare silica and on aluminized silica surfaces due to the electrostatic repulsion of dye molecules by $\mathrm{Al}-\mathrm{OH}$ groups in aluminized silica, and subsequent spectrophotometric quantification. The adsorbate molecule used for this study was malachite green. The difference between amount of malachite green retained on bare silica and

aluminized silica remained constant when the ratio of active sites on the support to malachite green molecules was not very different. The results indicated that the method presents sensitivity and selectivity for the determination of hydroxyl groups in aluminosilicate materials with high repeatability, making possible the quantification of $\mathrm{Al}-\mathrm{OH}$ in aluminosilicates without the use of sophisticated scientific instruments.

Keywords: Aluminized silica, malachite green, molecular absorption spectroscopy, Al-OH sites.

\section{INTRODUCTION}

For many years silica has been the main material used as chromatographic support for reversed-phase liquid chromatography (RP-LC). This wide use is associated with its pore structure; fully compatible with the mechanisms of chromatographic separation, producing stationary phases of greater efficiency in the resolution of several chemical species [1,2]. However, the limitation of silica-based stationary phases to a restricted $\mathrm{pH}$ range between 2 and 8 promoted a growing search for more stable inorganic oxides, mainly in alkaline medium, as supports for RP-LC [2-4]. Coating the silica with metal oxide layer was shown to be one of the most successful strategies in the preparation of chromatographic supports and led to the expansion of application field of RP-LC without loss in separation efficiencies [5-9]. More recently, silica coated with alumina has been presented as a chromatographic support that, in addition to greater chemical stability, results in a lower residual activity in separations by HPLC compared to coating with other metallic oxides [10-13]. Thus, polar solutes are not retained on the phases of aluminized silica as strongly as they are on silica coated with other metal oxides, such as zirconia $[14,15]$ and titania $[14,16]$. 
The alumina surface activity is related to concentration of hydroxyl groups attached to aluminum atoms on the aluminized silica surface and available to interact with the solutes in a chromatographic separation process. A high concentration of $\mathrm{Al}-\mathrm{OH}$ groups may lead to a reduction in the degree of surface coating due to the lower reactivity of these groups compared to silanols $(\mathrm{Si}-\mathrm{OH})$ in the preparation of reversed stationary phases, and also to an increase in residual activity, an undesirable interaction of acidic or basic compounds with these groups due to the amphoteric character of alumina [3,4,17]. Therefore, Al-OH group content on the surface of chromatographic supports is an important factor in obtaining both a stable and efficient support material for chromatographic purposes.

The determination of alumina activity on aluminized silica requires selective procedures to discriminate the hydroxyl groups from alumina from those of other oxides [18-21]. Most of the time this determination is performed directly by instrumental techniques, such as solid state nuclear magnetic resonance [19,21], infrared absorption spectroscopy [20,22], thermogravimetric analysis [22], etc. In all cases, it is necessary to use specific and high-purity reagents or high-cost instrumentation techniques that are difficult to access for most research laboratories. Therefore, the development of simpler and more easily accessible methods, without the need for specific reagents for the determination of the concentration of active surface groups of alumina, can lead to obtaining the desired information in a less expensive, faster and equally reliable manner. In this work, a simple method, possible to be performed in most chemistry laboratories, without the need for specific scientific instrumentation and/or expensive and expensive reagents, is proposed for the determination of active alumina groups in solid aluminized silica materials. The method is based on measuring the absorbance of a colored substance in solution before and after contact with an aluminacontaining adsorbent. The quantification of these active groups of alumina is carried out from the consequent discoloration of the solution and subsequent analysis by molecular absorption spectroscopy in the visible region.

\section{MATERIALS AND METHODS \\ Materials}

The chromatographic support used in the study was $5 \mu \mathrm{m}$ spherical Microsorb silica (Varian, Palo Alto, CA, USA). For the preparation of aluminized silica, isooctane analytical grade from Synth (Diadema, SP, Brazil), aluminum isopropoxide (99\%) and sodium bis(2-ethylhexyl)sulfosuccinate ( $>97 \%)$ from SigmaAldrich (São Paulo, SP, Brazil), and nitric acid (65\%) from Carlo Erba (Milan, Italy), were used. Sodium chloride PA and Malachite Green chloride (MG) of analytical grade purchased from Synth, and ultrapure water (resistivity > $18 \mathrm{M} \Omega \mathrm{cm}$ ) obtained from a Megapurity purification system (Billerica, MA, USA) were used in the study of adsorption isotherms. All solvents were individually filtered prior to use in a mobilephase filtration system employing nylon membranes with $0.22 \mu \mathrm{m}$ pore diameter from Millipore (São Paulo, SP, Brazil).

\section{Synthesis of aluminized silica particles by intra-micellar sol-gel process}

Alumina particles were produced by a sol-gel process within the sodium bis(2-ethylhexyl) sulfosuccinate micelles, $\mathrm{Na}(\mathrm{AOT})$, according to the method of Silveira et al. [11]. Briefly, $\mathrm{Na}(\mathrm{AOT})$ reversed micelles were produced in isooctane by adding $2.440 \mathrm{~g}$ of the surfactant to $36 \mathrm{~mL}$ of solvent under gentle and continuous stirring in a refluxing system at $100{ }^{\circ} \mathrm{C}$. Then $1.0 \mathrm{~mL}$ of ultrapure water and $0.250 \mathrm{~g}$ of aluminum isopropoxide were added to the $\mathrm{Na}(\mathrm{AOT})$ microemulsion, ensuring a ratio of surfactant/aluminum isopropoxide/water equal to 90.0:5.4:4.6 w/w/w. The reaction mixture was stirred for $2 \mathrm{~h}$ at $100{ }^{\circ} \mathrm{C}$. Subsequently, $900 \mu \mathrm{L}$ of $\mathrm{HNO}_{3}$ solution at $0.01 \mathrm{~mol} \mathrm{~L}^{-1}$ was added to the reaction medium. After peptization, the blend was aged for $24 \mathrm{~h}$ under constant stirring at $100{ }^{\circ} \mathrm{C}$. After this period the mixture was centrifuged at $2500 \mathrm{rpm}$ for $5 \mathrm{~min}$ and the alumina gel phase separated. 


\section{Technical Note}

dos Santos, A. L. R.; de Assunção, R. M. N; de Faria, A. M.

The alumina gel phase contained within the $\mathrm{Na}(\mathrm{AOT})$ reversed micelles was incorporated onto the surface of spherical silica particles using the layer-by-layer self-assembly technique. The alumina gel covered by a $\mathrm{Na}$ (AOT) layer was added to $20 \mathrm{~mL}$ of isooctane under stirring at $100^{\circ} \mathrm{C}$. After $2 \mathrm{~h}, 1.500 \mathrm{~g}$ of silica previously activated at $140{ }^{\circ} \mathrm{C}$ for $12 \mathrm{~h}$ was added to the reaction medium under reflux and kept under constant stirring for $24 \mathrm{~h}$. The surfactant layer on alumina gel acted as a 'glue' on the silica microspheres. After the reaction, the mixture was filtered on $0.22 \mu \mathrm{m}$ pore diameter nylon membranes and the solid material was calcined at $500{ }^{\circ} \mathrm{C}$ for $30 \mathrm{~min}$. The calcined material was washed sequentially with isooctane, toluene, isopropanol, methanol and ultrapure water. The silica particles were coated with a layer of alumina nanoparticles, and referred to as aluminized silica.

\section{Adsorption of malachite green on chromatographic supports Adsorption kinetics of malachite green}

To evaluate the adsorption kinetics of malachite green (MG) on silica and aluminized silica, $10 \mathrm{mg}$ of solid material was added to Eppendorf tubes containing $1 \mathrm{~mL}$ of an aqueous solution of MG at $100 \mathrm{mg} \mathrm{L}^{-1}$. Twelve aqueous solutions of $\mathrm{MG}$ in $0.010 \mathrm{~mol} \mathrm{~L}^{-1} \mathrm{NaCl}$ were prepared, adjusting $\mathrm{pH}$ to 5.00 before the study. The tubes were gently stirred at time intervals from 0 to $120 \mathrm{~min}$, maintaining a constant temperature of $27^{\circ} \mathrm{C}$. The absorbances of aliquots of the solutions, without solid material particles, were measured at $610 \mathrm{~nm}$ in a PerkinElmer UV-vis spectrophotometer, model Lambda 25 (Waltham, MA, USA) to determine the concentration of unretained MG on silica and aluminized silica. All experiments were performed in triplicate.

\section{Adsorption isotherms of malachite green}

The MG adsorption experiments on silica and aluminized silica were performed by adding $10 \mathrm{mg}$ of the solid materials to Eppendorf tubes containing $1.00 \mathrm{~mL}$ of $\mathrm{MG}$ solution in $0.01 \mathrm{~mol} \mathrm{~L}^{-1} \mathrm{NaCl}$ at $\mathrm{pH} 5$ over a range of concentrations of 0.1 to $300.0 \mathrm{mg} \mathrm{L}^{-1}$. The tubes were stirred gently for $5 \mathrm{~min}$ and then centrifuged at $3000 \mathrm{rpm}$ for $2 \mathrm{~min}$. Aliquots of supernatant were analyzed by molecular absorption spectroscopy in the visible region. All experiments were conducted at $27^{\circ} \mathrm{C}$ and performed in triplicate.

The experimental data obtained were used for the construction of adsorption isotherm curves of MG on bare silica and aluminized silica.

\section{Characterization of aluminized silica supports}

Specific surface area and porosity

The specific surface areas of silica and aluminized silica were determined by adsorption of nitrogen in the dry state, following the conventional BET (Brunauer, Emmet and Teller) method with an ASAP 2010 instrument from Micromeritics (Norcross, GA, USA).

\section{Scanning electron microscopy and energy-dispersive X-ray spectroscopy}

The alumina content on aluminized silica supports was determined by an X-ray energy dispersion spectrometer coupled to a TESCAN scanning electron microscope, model Vega3 (Brno, Czech Republic) at an acceleration voltage of $20 \mathrm{kV}$. The samples were prepared by fixing the silica and aluminized silica particles to conductive double-sided carbon tape, and coating with a gold film layer to increase the conductivity.

\section{RESULTS AND DISCUSSION \\ Characterization of aluminized silica support}

Scanning electron microscopy and energy-dispersive X-ray spectroscopy

The aluminum content in the aluminized silica support was determined by dispersive energy spectroscopy (EDS). Twelve distinct regions in three typical scanning electron micrographs of aluminized 
silica particles were analyzed by EDS and the obtained spectra were used for quantification of elements. Figure 1 shows a typical spectrum of one of the spectrally analyzed regions in aluminized silica micrographs.

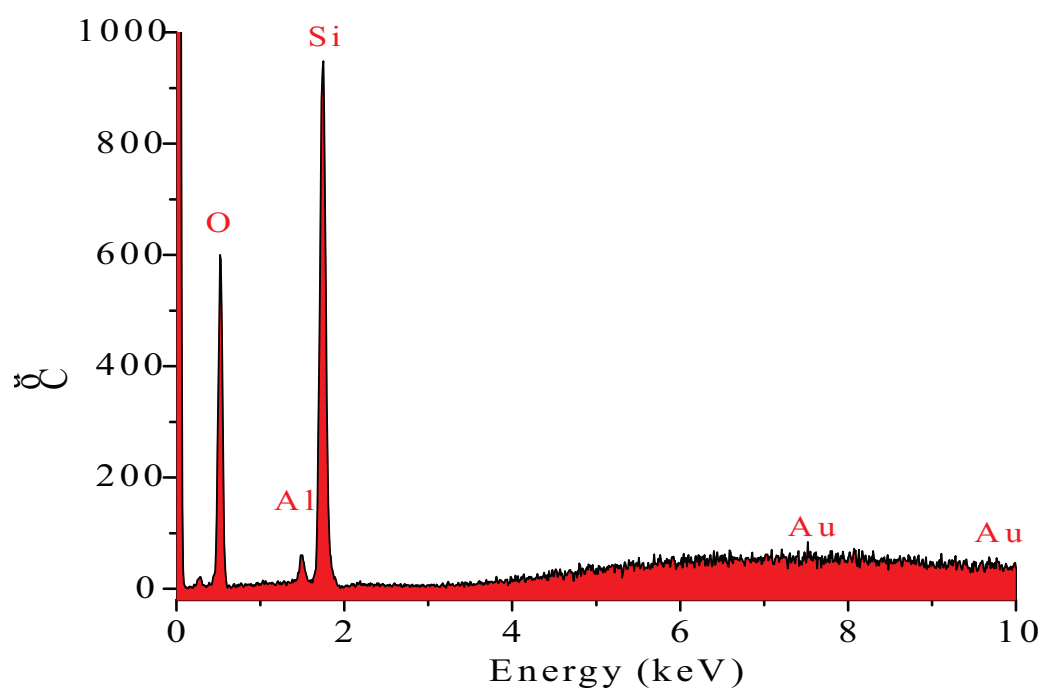

Figure 1. EDS spectrum of the aluminized silica sample

The EDS spectrum in Figure 1 indicates the presence of aluminum in aluminized silica support. The average aluminum content, as a mass percentage, obtained from twelve distinct regions in micrographs of aluminized silica particles was $1.61 \pm 0.14 \% \mathrm{w} / \mathrm{w}$. By converting this amount to the alumina content, the aluminized silica contained $3.05 \pm 0.27 \% \mathrm{w} / \mathrm{w}$ alumina on average. The small dispersion around the average alumina content indicates a regular distribution of metal oxide onto the silica surface. The relatively low content of alumina in the aluminized silica support is sufficient to increase its stability under alkaline mobile phase conditions without causing significant loss of separation efficiency. The activity of hydroxyl groups bound to the aluminum atoms was obtained by adsorption studies.

Micrographs of bare and aluminized silica are presented in Figure 2. It can be seen from the SEM images in Figure 2 that, in contrast to the bare silica particles, the aluminized silica has a layer of smaller alumina particles into the silica microsphere surface.

(a)

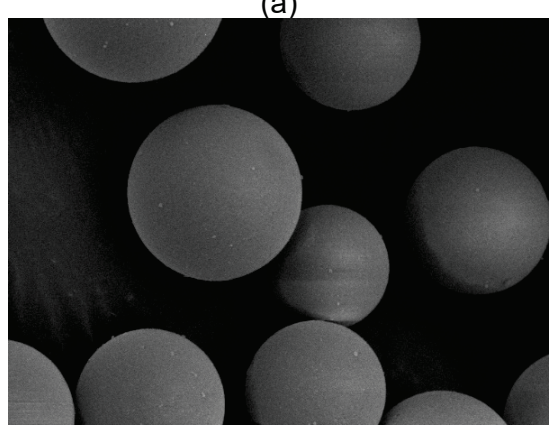

(b)

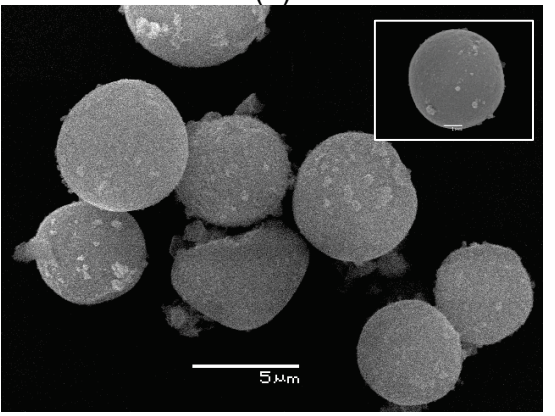

Figure 2. SEM images of (a) bare silica and (b) aluminized silica samples

\section{Specific surface area and porosity}

The specific surface area, pore volume and pore diameter of aluminized silica particles were determined by the physisorption technique. The morphological information of both silica and aluminized silica particles used in this study is presented in Table I. 
Table I. Morphological information of silica and aluminized silica particles

\begin{tabular}{lcccc}
\hline Material & $\begin{array}{c}\text { Specific surface } \\
\text { area }\left(\mathbf{m}^{2} \mathbf{~ g}^{-1}\right)\end{array}$ & $\begin{array}{c}\text { Pore volume } \\
\left(\mathbf{c m}^{3} \mathbf{~ g}^{-1}\right)\end{array}$ & $\begin{array}{c}\text { Pore diameter } \\
(\mathbf{n m})\end{array}$ & $\begin{array}{c}\text { Alumina } \\
\text { content }(\%)\end{array}$ \\
\hline Bare silica & $219 \pm 3$ & $0.67 \pm 0.02$ & $12.2 \pm 0.5$ & $<0.01$ \\
Aluminized silica & $212 \pm 7$ & $0.54 \pm 0.04$ & $10.5 \pm 0.6$ & $3.05 \pm 0.27$ \\
\hline
\end{tabular}

Despite the incorporation of about 3\% alumina onto the silica microparticles, the specific surface area of aluminized silica was not significantly reduced when compared to the bare silica. The deposition of alumina occurred onto the surface and into the pores of the silica particles, as there was a reduction of pore diameter and pore volume of the silica after incorporation of alumina. The active groups of silica, silanols $(\mathrm{Si}-\mathrm{OH})$, are distributed on the silica surface, but are mostly found inside the pores of mesoporous materials. Considering that the alumina is deposited on the silica by interaction/reaction with $\mathrm{Si}-\mathrm{OH}$ groups, a small reduction in the pore parameters of the aluminized silica was to be expected. This morphological information is important in determining the content of active $\mathrm{Al}-\mathrm{OH}$ groups on the aluminized silica surface because the molecule used in the adsorption study must be able to access all the pores of the chromatographic support.

\section{Studies of adsorption of malachite green onto aluminized silica particles}

The determination of $\mathrm{Al}-\mathrm{OH}$ content in the aluminized silica support was carried out by the study of interaction of malachite green (MG) onto the silica and aluminized silica supports, with subsequent analysis of the adsorbate solutions by absorption spectroscopy in the visible region. In this study, the $\mathrm{pH}$ of the solutions was adjusted to 5 , since the isoelectric point of alumina is at $\mathrm{pH} 8,23$ while that of silica is at about $\mathrm{pH} 2$ [24]. In slightly acidic medium ( $\mathrm{pH} 5$ ), the alumina and the MG will be positively charged [25], while the silica is negatively charged. Thus, the aluminized silica will electrostatically repel the MG, keeping the solution greenish in color. On the other hand, the negatively charged silica sites will attract the MG onto its surface, decolorizing the solution. The higher the content of $\mathrm{Al}-\mathrm{OH}$ sites on the aluminized silica support the lower the amount of MG retained on its surface, the less decolorization of the solution. It is important to emphasize that although we believe that the mechanism of ion exchange established between the adsorbate and the adsorbents is predominant, other interaction processes can also occur mainly under different dye concentrations in solution, in this way, we will use the term adsorption in this text to generalize the adsorbent-adsorbate interaction process. Figure 3 shows the distribution of MG species according to the $\mathrm{pH}$ of the aqueous solution. 


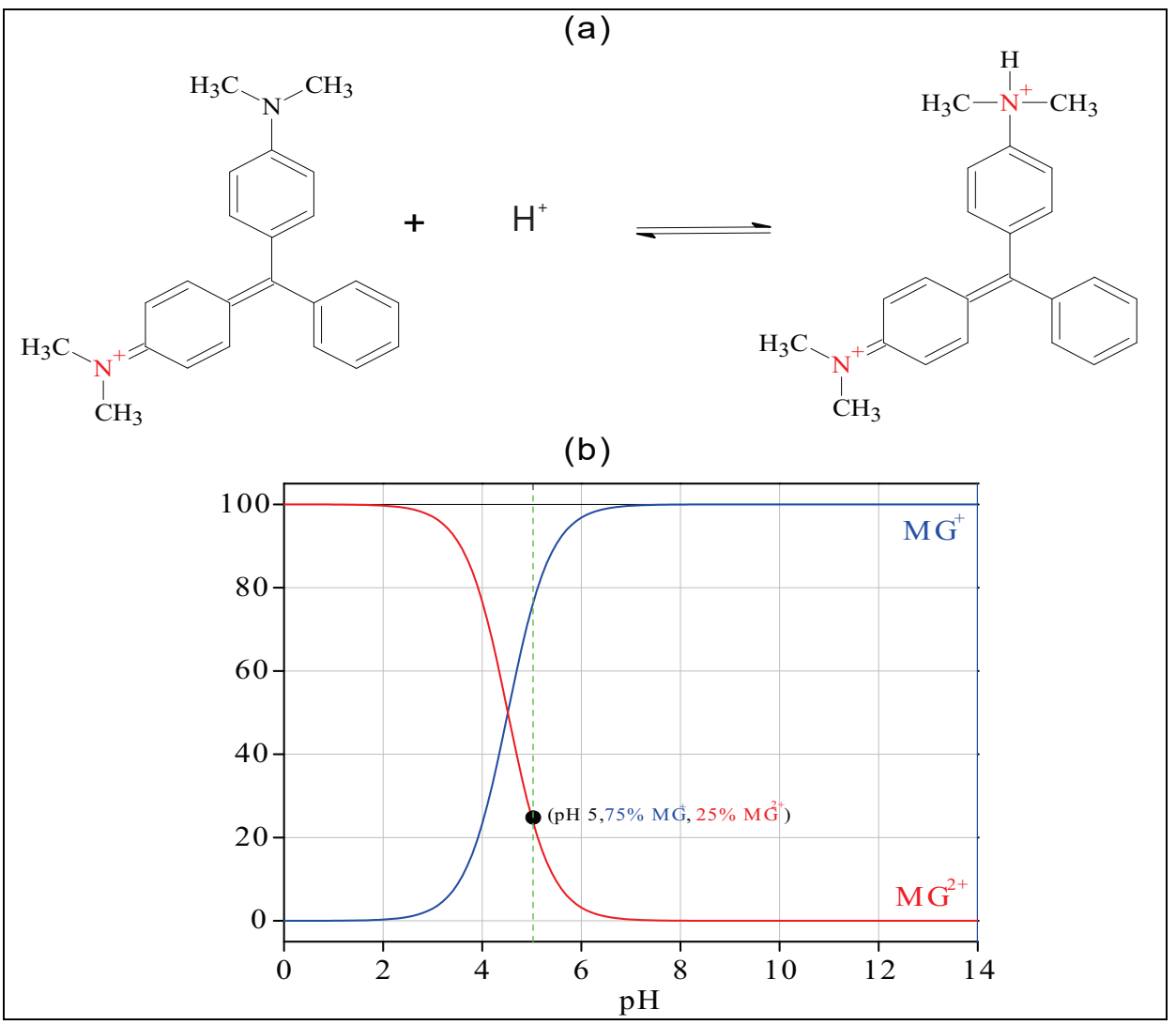

Figure 3. (a) Chemical equilibrium of malachite green in aqueous solution. (b) Graph of distribution of malachite green (MG) species in aqueous solution as a function of $\mathrm{pH}$ [26].

The concentration of MG in solution that was not retained on silica or on aluminized silica was determined by constructing an analytical curve for the malachite green. The linear equation, obtained by linear regression of absorbance data for $\mathrm{MG}$ solutions in $0.01 \mathrm{~mol} \mathrm{~L}^{-1} \mathrm{NaCl}$ in the concentration range of 0.005 to $5.000 \mathrm{mg}$ $L^{-1}$, was: $A=0.1503 C_{M G}-0.0052$, with coefficient of determination $R^{2}=0.9997$.

\section{Adsorption kinetics of malachite green on aluminized silica}

Adsorption is a multi-stage process that involves transporting MG molecules from aqueous phase to the surface of aluminized silica particles, followed by the diffusion of molecules dissolved within the pores of the support. The adsorption kinetics represent the change in ratio with time of the amount of free MG to that adsorbed on the aluminized silica. The effect of time on the adsorption of MG on aluminized silica support is shown in Figure 4.
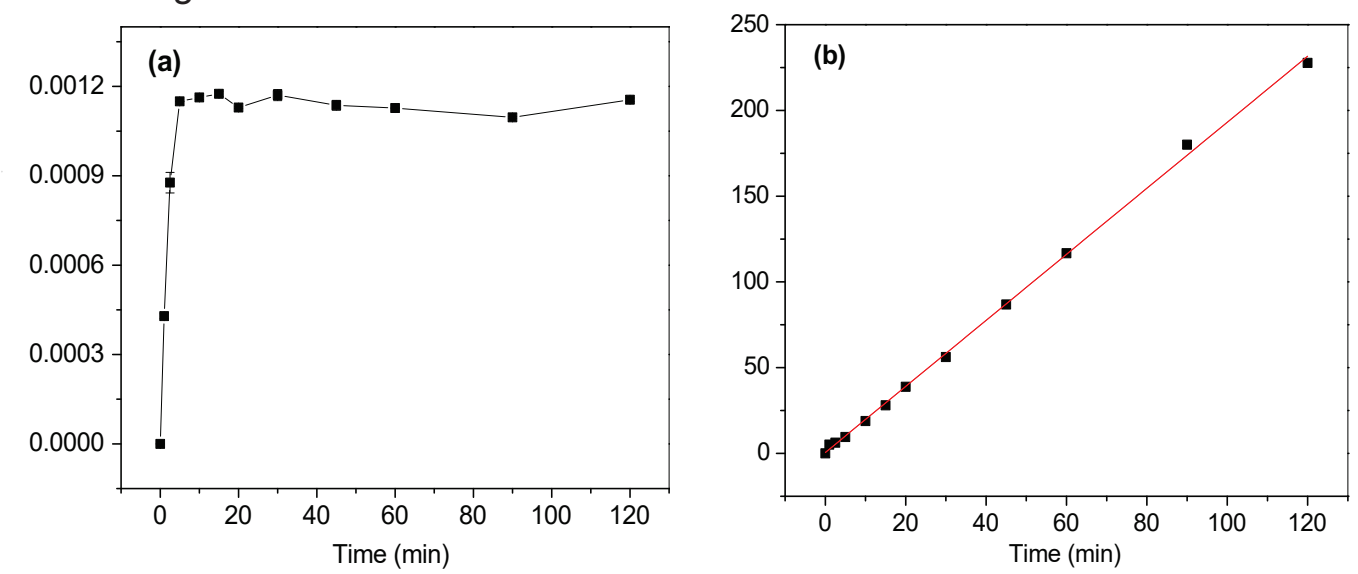

Figure 4. (a) Curve for adsorption kinetics of malachite green on aluminized silica support. (b) Pseudo-second-order kinetic model for the adsorption of malachite green on aluminized silica 


\section{Technical Note}

From Figure 4 the adsorption of MG on aluminized silica was initially very fast, reaching equilibrium in around $5 \mathrm{~min}$, from when it remained practically constant. The mechanism involved in the kinetics for the removal of $\mathrm{MG}$ from the solution by the aluminized silica support was investigated by applying the experimental data in the equation for the pseudo-first-order (Equation 1), pseudo-second-order (Equation 2) and intraparticle diffusion kinetic models [27] (Equation 3).

$$
\log \left(q_{e}-q_{t}\right)=\log \left(q_{e}\right)-k_{1} t / 2.303
$$

qe $\left(\right.$ meq $\left.\mathrm{g}^{-1}\right)$ and $q t$ (meq $\left.\mathrm{g}^{-1}\right)$ are the amounts of MG adsorbed per unit mass of aluminized silica at equilibrium and at any time $t$, respectively; $\left(\mathrm{min}^{-1}\right)$ is the constant of pseudo-first-order adsorption rate.

$$
\mathrm{t} / \mathrm{q}_{\mathrm{t}}=1 /\left(\mathrm{k}_{2} \mathrm{q}_{\mathrm{e}}^{2}\right)+\mathrm{t} / \mathrm{q}_{\mathrm{e}}
$$

qe $\left(\right.$ meq $\mathrm{g}^{-1}$ ) and $q$ t (meq $\mathrm{g}^{-1}$ ) are the amounts of MG adsorbed per unit mass of aluminized silica at equilibrium and at any time $t$, respectively; $\left(\mathrm{g} \mathrm{meq}^{-1} \mathrm{~min}^{-1}\right)$ is the constant of pseudo-second-order adsorption rate, which is obtained by the angular coefficient of the curve in Figure 3(b).

$$
\mathrm{q}_{\mathrm{t}}=\mathrm{k}_{\mathrm{i}} \mathrm{t}^{1 / 2}+\mathrm{C}
$$

$q t\left(\mathrm{meq} \mathrm{g}^{-1}\right)$ is the amount of $\mathrm{MG}$ adsorbed per unit mass of aluminized silica at any time $t$; $\left(\mathrm{meq}^{-1}\right.$ $\min ^{-0.5}$ ) is the constant of intraparticle diffusion rate and $C$ is the coefficient of linear regression.

A better fit with the data occurs with the pseudo-second-order kinetic model for the MG adsorption on aluminized silica, confirmed by the coefficient of determination, $\mathrm{R}^{2}=0.999$, presenting it as the appropriate kinetic model and indicating the existence of chemisorption in the studied process. The chemisorption process can be explained by an ion exchange process between the positively charged MG and the deprotonated silanol groups in aluminized silica, under the $\mathrm{pH}$ condition studied. The rate constant for MG adsorption was $3.12 \times 10^{3} \mathrm{~g} \mathrm{meq}^{-1} \mathrm{~min}^{-1}$, which means a rapid adsorption rate on aluminized silica. The value of qe calculated from the model was $1.14 \times 10^{-3} \mathrm{meq} \mathrm{g}^{-1}$, while the experimental qe was $1.15 \times 10^{-3}$ meq $\mathrm{g}^{-1}$. Thus, the best fit to the pseudo-second-order mechanism can be confirmed by the proximity of the qe value determined experimentally and that calculated from the model equation. Table II summarizes the

\begin{tabular}{|c|c|c|c|c|}
\hline \multicolumn{5}{|l|}{ Pseudo-first-order } \\
\hline$k_{1}\left(\min ^{-1}\right)$ & $\mathrm{q}_{\mathrm{e}}$ calc $\left(\right.$ meq g ${ }^{-1}$ ) & $q_{e} \exp \left(m e q g^{-1}\right)$ & & $r$ \\
\hline $5.14 \times 10^{-3}$ & $5.749 \times 10^{-3}$ & $1.14 \times 10^{-3}$ & & 0.399 \\
\hline \multicolumn{5}{|c|}{ Pseudo-second-order } \\
\hline$k_{2}\left(g \mathrm{meq}^{-1} \mathrm{~min}^{-1}\right)$ & $\mathrm{q}_{\mathrm{e}}$ calc $\left(\right.$ meq $\left.\mathrm{g}^{-1}\right)$ & $q_{e} \exp \left(m e q g^{-1}\right)$ & $\mathbf{r}$ & $\mathrm{h}\left(\mathrm{meq} \mathrm{g}^{-1} \mathrm{~min}^{-1}\right)$ \\
\hline $3.12 \times 10^{3}$ & $1.14 \times 10^{-3}$ & $1.15 \times 10^{-3}$ & 0.999 & $4.04 \times 10^{-3}$ \\
\hline \multicolumn{5}{|c|}{ Intraparticle diffusion } \\
\hline$k_{\text {dif }}\left(\right.$ meq g $\left.^{-1} \min ^{-0,5}\right)$ & & C & & $\mathbf{r}$ \\
\hline $6.62 \times 10^{-5}$ & & 0.299 & & 0.613 \\
\hline
\end{tabular}
calculated parameters for the kinetic models.

Table II. Kinetic parameters for the interaction of malachite green with aluminized silica particles 


\section{Isotherm models for the adsorption of malachite green on bare and aluminized silica}

The adsorption isotherms describe the equilibrium between adsorbent and adsorbate (solute) considering the ratio between the amount of solute adsorbed and that remaining in solution at a fixed temperature. Since the adsorption phenomenon depends on the interactions between the surface of the adsorbent and the adsorbed species, it is possible to use information about the adsorption process to identify the surface modification of an adsorbent [28].

To investigate the surface modification of silica after incorporation of nanoparticles of alumina, the adsorption isotherms of MG on bare silica and on aluminized silica were evaluated using the isotherm models of Langmuir, Freundlich and Sips, Equations 4-6, respectively.

$$
\begin{aligned}
& \mathrm{q}_{\mathrm{e}}=\mathrm{Q}_{\max } \mathrm{K}_{\mathrm{L}} \mathrm{C}_{\mathrm{e}} / 1+\mathrm{K}_{\mathrm{L}} \mathrm{C}_{\mathrm{e}} \\
& \mathrm{q}_{\mathrm{e}}=\mathrm{K}_{\mathrm{F}} \mathrm{C}_{\mathrm{e}}^{1 / \mathrm{nF}} \\
& \mathrm{q}_{\mathrm{e}}=\mathrm{Q}_{\max } \mathrm{K}_{\mathrm{s}} \mathrm{C}_{\mathrm{e}}^{\mathrm{ns}} / 1+\mathrm{K}_{\mathrm{s}} \mathrm{C}_{\mathrm{e}}^{\mathrm{ns}}
\end{aligned}
$$

where: $\mathrm{q}_{\mathrm{e}}=$ amount of adsorbate per unit mass of adsorbent at equilibrium ( $\left.\mathrm{meq} \mathrm{g}^{-1}\right) ; \mathrm{Ce}=$ concentration of adsorbate at equilibrium (meq $\mathrm{L}^{-1}$ ); $\mathrm{Q}_{\max }=$ maximum adsorption capacity (meq g ${ }^{-1}$ ); $\mathrm{K}_{\mathrm{L}}=$ Langmuir constant related to the free energy of adsorption $\left(L \mathrm{meq}^{-1}\right) ; n_{F}=$ Heterogeneity factor, which indicates adsorption intensity; $\mathrm{K}_{\mathrm{F}}=$ Freundlich constant, which indicate adsorption capacity; $\mathrm{n}_{\mathrm{s}}=$ Dimensionless heterogeneity factor; $K_{s}=$ Sips equilibrium constant related to the free energy of adsorption $\left(L\right.$ meq $\left.^{-1}\right)$

The Langmuir and Freundlich isotherm models are the most used in the evaluation of adsorption processes. In the first case, the adsorption of a monolayer of the adsorbate occurs on the surface of the adsorbent due to the presence of homogeneous sites of the same energy. This process is characterized by Equation 4, developed from the equilibrium condition of the adsorption process. Freundlich's isotherm (Equation 5), on the other hand, admits a multilayer adsorption process and predicts the heterogeneity of adsorption sites on the adsorbent surface. The application of these two models to the MG adsorption data on bare silica and aluminized silica can be observed in Figure 5 and Table III.
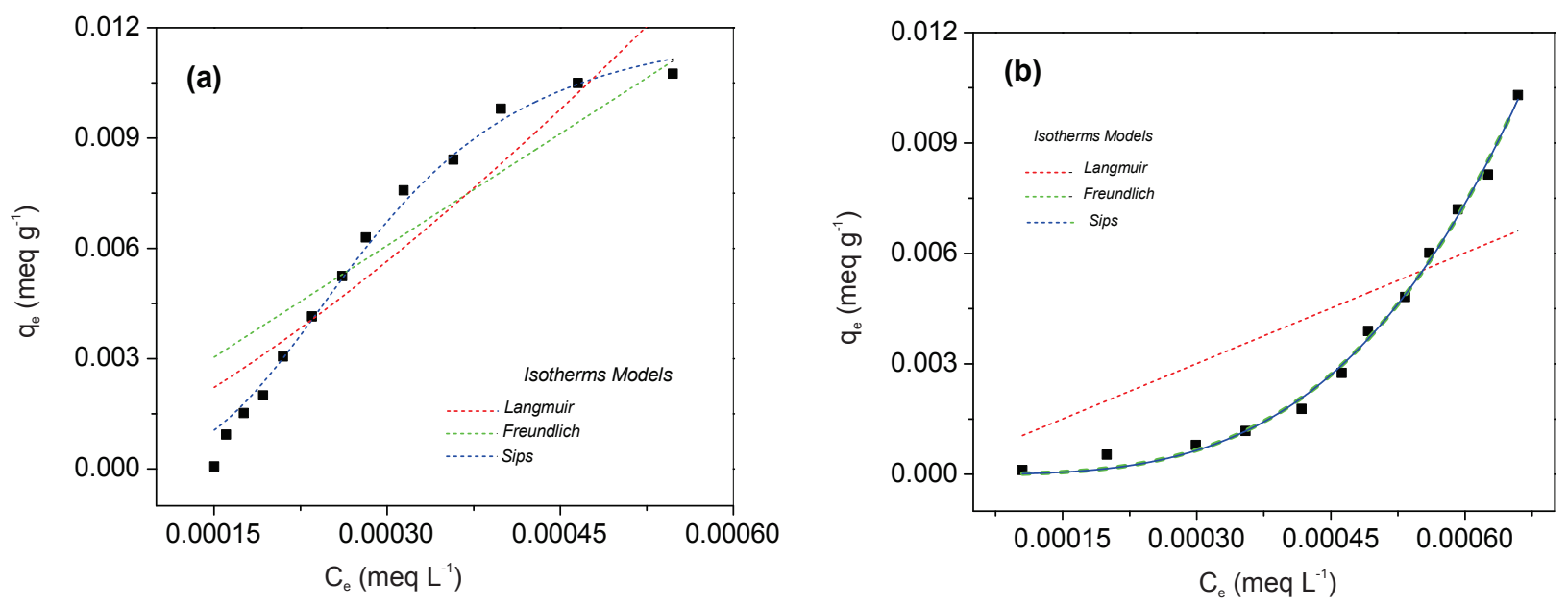

Figure 5. Adsorption isotherm models of malachite green on (a) bare silica and (b) isotherm models of malachite green on aluminized silica in $0.01 \mathrm{~mol} \mathrm{~L}^{-1} \mathrm{NaCl}$ solution at $\mathrm{pH} 5.00$

The profile of experimental data shown in Figure 5 (a) is similar to the pattern of the predicted graph in the Langmuir model. In this model, the graph is characterized by the presence of a plateau at which saturation is observed, i.e. a molecule occupies the site and no additional adsorption occurs [29]. However, 
the non-linear regression of the experimental data in relation to the Langmuir and Freundlich models was not adequate, since the adjusted determination coefficient deviates from 1 , indicating a poor fit of experimental data in relation to the predicted curves in each model.

The application of the Freundlich model in the study of MG adsorption on aluminized silica, Figure 5(b), shows that the adsorption process is unfavorable, in this condition, since the convex profile of the curve indicates low dye removal capacity within the range of MG concentrations studied, 0.05 to $0.15 \mathrm{mg} \mathrm{L}^{-1}$, for the quantification of $\mathrm{Al}-\mathrm{OH}$ groups. The value of the ratio $1 / \mathrm{n}$ is equal to 3.475 , which indicates heterogeneity of the superficial sites and weaker interaction between the adsorbent and adsorbate compared to the adsorbate-adsorbate interaction (Table III).

It is important to note that the adjusted determination coefficient shows a value of 0.995 , indicating good fit with the data on adsorption of $M G$ on aluminized silica using the Freundlich model. In the same experimental conditions, the bare silica and the aluminized silica showed marked differences in the mechanism of adsorption of MG. The Langmuir and Freundlich models are based on the hypothesis of physical adsorption and therefore present a poor description of the system under study, which should be based models that may consider greater complexity as chemical reactions involved in adsorption processes. To better evaluate the adsorption differences between the two adsorbents, a model with three parameters was applied: Sips model. The results show a good fit with experimental data and are presented in Table III.

Table III. Comparison of the parameters of isotherm models of adsorption of malachite green on bare silica and aluminized silica

\begin{tabular}{|c|c|c|}
\hline \multicolumn{3}{|c|}{ Langmuir } \\
\hline Parameters & Bare Silica & Aluminized Silica \\
\hline$Q_{\max }\left(\right.$ meq g $\left.^{-1}\right)$ & 73.644 & 106.520 \\
\hline $\mathrm{K}_{\mathrm{L}}\left(\mathrm{L} \mathrm{meq}^{-1}\right)$ & 0.275 & 0.094 \\
\hline $\mathrm{R}^{2}$ adjusted & 0.800 & 0.617 \\
\hline \multicolumn{3}{|c|}{ Freundlich } \\
\hline Parameters & Bare Silica & Aluminized Silica \\
\hline $\mathrm{K}_{\mathrm{F}}\left(\mathrm{meq}^{1-(1 / n)}\left(\mathrm{g}^{-1}\right) \mathrm{L}^{1 / n}\right)$ & 324.653 & 1.154 \\
\hline $1 / n_{F}$ & 1.351 & 3.475 \\
\hline $\mathrm{R}^{2}$ adjusted & 0.862 & 0.995 \\
\hline \multicolumn{3}{|c|}{ Sips } \\
\hline Parameters & Bare Silica & Aluminized Silica \\
\hline$Q_{\max }\left(\operatorname{meq~g} g^{-1}\right)$ & 0.012 & 0.469 \\
\hline $\mathrm{n}_{\mathrm{s}}$ & 3.711 & 3.519 \\
\hline $\mathrm{K}_{\mathrm{s}}\left(\mathrm{L} \mathrm{meq}^{-1}\right)^{-\mathrm{n}}$ & $1.488 \times 10^{13}$ & 3.465 \\
\hline $\mathrm{R}^{2}$ adjusted & 0.987 & 0.994 \\
\hline
\end{tabular}

The Sips isotherm is an empirical equation that presents a combination of Langmuir and Freundlich isotherms. The equation was developed by considering the equilibrium involved in the adsorption process. High $\mathrm{K}_{\mathrm{s}}$ values indicate a very favorable adsorption process, in contrast to low $\mathrm{K}_{\mathrm{s}}$ values. The best results observed for fit of experimental curves for bare silica and aluminized silica were obtained using the Sips model (Table III). The $\mathrm{K}_{\mathrm{s}}$ value for bare silica, $1.488 \times 10^{13}$, indicates that the MG adsorption process on bare silica was extremely favorable, in contrast to the value 3.465 found for aluminized silica. For the aluminized silica, when applying the Sips model, which is a combination of Langmuir and Freundlich isotherms, an extremely high value is observed for the maximum adsorption capacity. This value is a result 
of the tendency of Sips model to follow the Freundlich model, so this value indicates the theoretical condition of infinite adsorption and should not be compared to the real value of maximum adsorption capacity on the silica. The results show significant differences in the adsorption process due to the incorporation of alumina nanoparticles on silica surface. To quantify the experimental modification, the adsorption of MG was evaluated at low concentrations, in which the adsorption of MG is linear, using the initial concentration of the solutions to evaluate the amount adsorbed.

\section{Quantification of Al-OH sites on aluminized silica}

To understand the adsorption behavior of MG on the aluminized silica surface, an adsorption isotherm was constructed. To verify the effect of MG adsorption on aluminized silica, the MG adsorption on bare silica, which does not possess $\mathrm{Al}-\mathrm{OH}$ sites on its surface, was compared with it. The adsorption isotherms of malachite green on both chromatographic supports are shown in Figure 5.

It can be observed that the adsorption of MG presented a distinct behavior on bare silica compared to that on aluminized silica support. The adsorption of MG on bare silica resulted in a graph with concave curvature, Figure 5(a). This condition implies a favorable adsorption of relatively high amounts of MG, even at low concentration levels. This isotherm is considered L-type because it has an initial curvature facing down due to the decrease in the availability of active sites on the adsorbent [30]. For the adsorption of MG on aluminized silica the graph resulted in a convex curvature, Figure 5(b), which implies unfavorable MG adsorption on aluminized silica, due to its low capacity for removal of MG from solution at low concentrations. Convex adsorption curves are considered S-type isotherms, in which adsorbate-adsorbent interactions are weaker than adsorbate-adsorbate or adsorbent-solvent interactions [30].

This difference between the behavior of MG adsorption on bare silica and on aluminized silica can be therefore associated with the presence of $\mathrm{Al}-\mathrm{OH}$ sites on the latter adsorbent. It was observed that for the adsorption study using intermediate MG concentrations, conditions in which the number of active sites of the supports is not much higher than that of MG molecules, and that the number of MG molecules is not significantly higher than that of sites, there is a linear behavior between the amount of MG adsorbed on the supports and the initial MG concentration in solution. Figure 6 shows the adsorption curves obtained for MG on bare silica and aluminized silica at these MG concentrations.

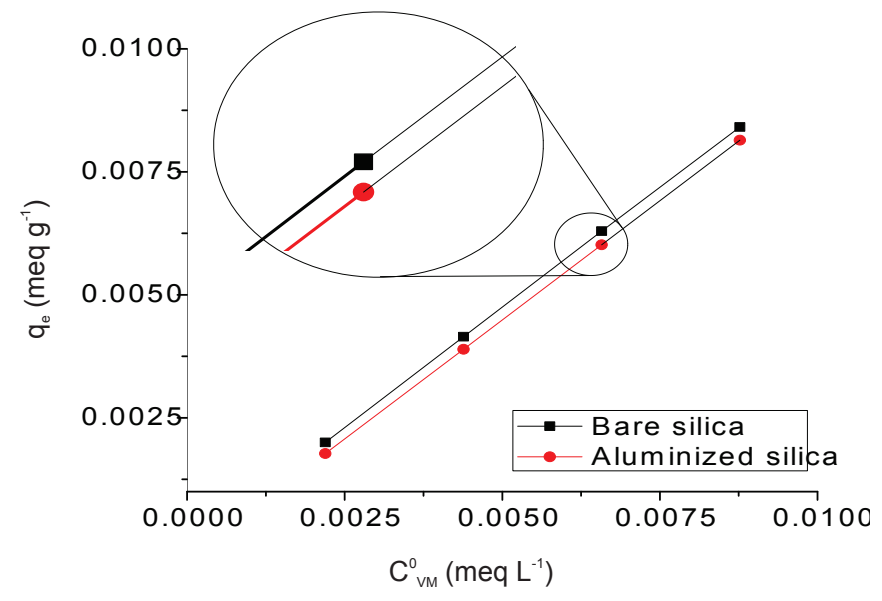

Figure 6. Adsorption curves of MG on bare silica and aluminized silica in a narrow range of $M G$ concentration in solution. In detail, the difference in amount of MG adsorbed on the two supports under the same experimental conditions.

According to Figure 6, the amounts of MG adsorbed on bare silica and on aluminized silica at intermediate MG concentrations in solution exhibit linear behavior. Furthermore, the difference between the amount of MG adsorbed on bare silica and the amount adsorbed on aluminized silica remains constant 


\section{Technical Note}

within the range of concentrations evaluated. This difference may be related, by approximation, to inactivation of silica adsorption sites occupied by Al-OH groups. The lower amount of MG adsorbed on aluminized silica may be related to the electrostatic repulsion suffered by the (positively charged) MG molecules by the (positively charged) $\mathrm{Al}-\mathrm{OH}$ groups. Therefore, the difference of adsorbed MG on these two materials can be correlated to the number of active $\mathrm{Al}-\mathrm{OH}$ groups on the aluminized silica surface. According to the data extracted from the curves in Figure 6, the mean difference between the amount of MG adsorbed on bare silica and on aluminized silica was $0.122 \pm 0.003 \mathrm{mg} \mathrm{g}^{-1}$ for the different initial concentrations of $M G$ in solution. Considering a molar mass of $M G$ of $329.458 \mathrm{~g} \mathrm{~mol}^{-1}$, the mean number of moles of MG that adsorbed further on bare silica was $0.371 \pm 0.003 \mu \mathrm{mol} \mathrm{g}^{-1}$. Considering also that the ratio between $\mathrm{MG}$ and $\mathrm{Al}-\mathrm{OH}$ is 1.25:1 (considering that at $\mathrm{pH} 525 \%$ of $\mathrm{MG}$ species are doubly charged cations) in the electrostatic repulsion between the adsorbate and adsorbent, and that the specific surface area of aluminized silica is $204 \mathrm{~m}^{2} \mathrm{~g}^{-1}$, the concentration of $\mathrm{Al}-\mathrm{OH}$ on the aluminized silica surface was 2.28 $\pm 0.02 \mathrm{nmol} \mathrm{m}{ }^{-2}$. The obtained value is significantly low, which implies that aluminized silica has a low activity of $\mathrm{Al}-\mathrm{OH}$ sites on its surface and pores, with alumina being predominantly incorporated in the form of $\mathrm{Al}_{2} \mathrm{O}_{3}$ particles. These values refer to nine experiments carried out independently, which are, therefore, authentic replicates with a low dispersion of values around the mean. The low dispersion can be attributed to the repeatability of the method to quantify the content of active alumina sites on an aluminized silica surface, which makes the method an interesting alternative to the more sophisticated instrumental techniques to perform this type of measurement.

It is also important to emphasize that knowledge of the content of $\mathrm{Al}-\mathrm{OH}$ sites on the surface of a chromatographic support is an important factor for the preparation of stationary phases for high performance liquid chromatography. The presence of very high numbers of $\mathrm{Al}-\mathrm{OH}$ sites may lead to greater difficulty in modification of the support with organic groups to produce reversed stationary phases and/or the presence of amphoteric residual sites, which may interact undesirably with basic and acidic solutes.

\section{CONCLUSIONS}

A new method was successfully employed to determine the content of $\mathrm{Al}-\mathrm{OH}$ groups in a solid aluminosilicate material from the differential adsorption of malachite green, and analysis of the adsorbate solution by molecular absorption spectroscopy. The method, besides being simple and easy to perform, does not require sophisticated instrumentation or expensive and specific reagents to perform a selective determination of hydroxyl groups in aluminized silica materials. The method presented excellent repeatability, showing itself as an alternative to the conventional, expensive and difficult-to-access methods. The proposed method is suitable for aluminosilicate-based materials and the experimental conditions must be strictly controlled so that the adsorbate adsorbs differentially with the groups to be determined. The aluminized silica support studied had an $\mathrm{Al}-\mathrm{OH}$ active group content suitable for chromatographic purposes, according to the method. The low number of $\mathrm{Al}-\mathrm{OH}$ groups obtained does not significantly affect the subsequent modification with organic clusters to produce reversed phases and does not imply the presence of residual groups after this modification, which could hinder the chromatographic separation of acidic or basic compounds.

Manuscript received March 19, 2018; revised manuscript received May 26, 2018; accepted June 25, 2018; published online October 3, 2018. 


\section{REFERENCES}

1. Stella, C.; Rudaz, S.; Veuthey, J. L.; Tchapla, A. Chromatographia, 2001, 53, pp S113-S131.

2. Qiu, H.; Liang, X.; Sun, M., Jiang, S. Anal. Bioanal. Chem., 2011, 399, pp 3307-3322.

3. Nawrocki, J.; Dunlap, C.; Li, J.; Zhao, J.; McNeff, C. V.; McCormick, A.; Carr, P. W. J. Chromatogr. A, 2004, 1028, pp 31-62.

4. Claessens, H. A.; van Straten, M. A. J. Chromatogr. A, 2004, 1060, pp 23-41.

5. Silva, C. R.; Airoldi, C.; Collins, K. E.; Collins, C. H. J. Chromatogr. A, 2008, 1191, pp 90-98.

6. Collins, C. H.; Silva, C. R.; Faria, A. M.; Collins, K. E.; Jardim, I. C. S. F. J. Braz. Chem. Soc., 2009, 20, pp 604-612.

7. Silva, C. G. A.; Collins, C. H.; Lesellier, E.; West, C. J. Chromatogr. A, 2013, 1315, pp 176-187.

8. Ge, J.; Zhao, L.; Chen, L. R.; Shi, Y. P. J. Chromatogr. Sci., 2010, 48, pp 29-34.

9. Amparo, M. R.; Marques, F. A.; Faria, A. M. J. Braz. Chem. Soc., 2013, 24, pp 1512-1519.

10. Goraieb, K.; Bueno, M. I. M. S.; Collins, C. H.; Collins, K. E. Quím. Nova, 2013, 36, pp 1131-1138.

11. Silveira, J. L.; Dib, S. R.; Faria, A. M. Anal. Sci., 2014, 30, pp 285-291.

12. Song, Z.; Wu, D.; Ding, K.; Guan, Y. J. Chromatogr. A, 2016, 1433, pp 85-89.

13. Faramawy, S.; El-Naggar, A. Y.; El-Fadly, A. M.; El-Sabagh, S. M.; Ibrahim, A. A. Arab. J. Chem., 2016, 9, pp S765-S775.

14. Borges, E. M.; Collins, C. H. J. Chromatogr. A, 2011, 1218, pp 4378-4388.

15. Silva, C. G. A.; Collins, C. H. J. Chromatogr. A, 2012, 1232, pp 248-256.

16. Faria, A. M.; Collins, K. E.; Collins, C. H. Chromatographia, 2008, 67, pp 357-363.

17. Nawrocki, J.; Dunlap, C.; McCormick, A.; Carr, P. W. J. Chromatogr. A, 2004, 1028, pp 1-30.

18. Zhao, Q.; Chen, W. H.; Huang, S. J.; Wu, Y. C.; Lee, H. K.; Liu, S. B. J. Phys. Chem. B, 2008, 106, pp 4462-4469.

19. Fry, R. A.; Tsomaia, N.; Pantano, C. G.; Mueller, K. T. J. Am. Chem. Soc., 2003, 125, pp 2378-2379.

20. Hensen, E. J. M.; Poduval, D. G.; Ligthart, D. A. J. M.; van Veen, J. A. R.; Rigutto, M. S. J. Phys. Chem. C, 2010, 114, pp 8363-8374.

21. Malfait W. J.; Xue, X. Geochim. Cosmochim. Acta, 2010, 74, pp 719-737.

22. Gallas, J. P.; Goupil, J. M.; Vimont, A.; Lavalley, J. C.; Gil, B.; Gilson, J. P.; Miserque, O. Langmuir, 2009, 25, pp 5825-5834.

23. Gulicovski, J. J.; Cerovic, L. S.; Milonjic, S. K. Mat. Manuf. Process., 2008, 23, pp 615-619.

24. Kokunesoski, M.; Gulicovski, J.; Matovic, B.; Logar, M.; Milonjic, S. K.; Babic, B. Mat. Chem. Phys., 2010, 124, pp 1248-1252.

25. Hameed, B. H.; El-Khaiary, M. I. J. Hazard. Mat., 2008, 153, pp 701-708.

26. https://chemicalize.com/[Accessed 12 December 2017]

27. Ho, Y. S.; McKay, G. Process. Biochem., 1999, 34, pp 451-465.

28. Belhachemi, M.; Addoun, F. Appl. Water Sci., 2011, 1, pp 111-117.

29. Foo, K. Y.; Hameed, B. H. Chem. Eng. J., 2010, 156, pp 2-10.

30. Dias Filho, N. L.; Carmo, D. R. Adsorption at Silica, Alumina and Related Surfaces. In: Hubbard, A. T. (Ed.). Encyclopedia of Surface and Colloid Science. Marcel Dekker, New York, N. J., 2004, pp 120. 